\title{
MECANISMOS CIRÚRGICOS DE CONTROLE DO DIABETES MELLITUS TIPO2 APÓS CIRURGIA BARIÁTRICA
}

\author{
MECHANISMS OF SURGICAL CONTROL FOR TYPE 2 DIABETES MELLITUS AFTER \\ BARIATRIC SURGERY
}

\author{
Marcus Vinicius Dantas de Campos Martins, TCBC - RJ'; \\ Antônio Augusto Peixoto de Souza, TCBC - RJ²
}

\section{INTRODUÇÃO}

O diabetes mellitus do tipo 2 é uma doença epidêmica, afetando mais de 150 milhões de pessoas em todo o mundo e com expectativa de duplicação nas primeiras décadas desse milênio ${ }^{1}$. Desse total, cerca de $90 \%$ estão acima do peso ou são obesos ${ }^{2}$ e o grau de obesidade é o principal fator preditor da ocorrência de diabetes em uma população ${ }^{3}$.

Os graus mais acentuados de obesidade associamse ainda, além do diabetes, com outras doenças como a hipertensão arterial sistêmica e as dislipidemias. A cirurgia bariátrica é atualmente o método mais eficiente por ocasionar a perda de peso e controlar as co-morbidades nesses pacientes acentuadamente obesos.

Entre as opções cirúrgicas disponíveis, o bypass gástrico em Y de Roux é o mais empregado. Esse procedimento cria uma pequena câmara gástrica proximal com exclusão de grande parte do estômago, de todo o duodeno e de parte do jejuno proximal do trânsito alimentar. O jejuno é dividido cerca de 50 centímetros após o ângulo de Treitz. A alça de jejuno distal é então anastomosada ao pequeno reservatório gástrico e uma jejuno-jenonostomia realizada a cerca de um metro da gastrojejunostomia.

Os mecanismos responsáveis pela perda de peso e pelo controle das doenças associadas incluem não só o grande efeito restritivo e algum grau de má-absorção que essa operação produz, mas também efeitos hormonais responsáveis pelo controle do apetite e velocidade do trânsito intestinal do alimento ${ }^{4}$.

As cirurgias bariátricas são extremamente eficientes no controle de algumas doenças associadas, especialmente o diabetes tipo II. A resolução do diabetes acontece precocemente após esse tipo de operação, antes mesmo que ocorra grande perda de peso $^{5}$. Tal fato pode ser explicado pelo efeito endócrino que esse procedimento produz, mesmo no período pós-operatório mais precoce ${ }^{6}$.

Crescente interesse nos mecanismos responsáveis por essa dramática e rápida melhora do diabetes após o tratamento cirúrgico são atualmente motivo de inúmeras pesquisas, não só pela possibilidade de desenvolvimento de um procedimento cirúrgico capaz de controlar o diabetes, mas também pelo interesse na pesquisa de novos medicamentos que possam produzir os mesmos mecanismos que a cirurgia proporciona.

\section{PATOGÊNESE DO DIABETES MELLITUS TIPO 2}

A discussão da patogênese do diabetes mellitus do tipo 2 deve começar com uma breve revisão dos mecanismos envolvidos na manutenção da homeostase glicêmica. Aproximadamente $50 \%$ de toda glicose disponível é utilizada no cérebro e não depende da ação da insulina ${ }^{7}$. Outros $25 \%$ são utilizados no território esplâncnico, e também são independentes da insulina. Os $25 \%$ restantes são usados em tecidos insulino-dependentes como o músculo e, em menor extensão, o tecido adiposo.

Aproximadamente $85 \%$ da produção endógena de glicose está associada ao fígado, sendo a glicogenólise e a gliconeogênese igualmente responsáveis por essa produção.

Após a alimentação, a elevação dos níveis plasmáticos de glicose estimulam a liberação de insulina. A hiperglicemia associada a hiperinsulinemia estimula a utilização da glicose pelos tecidos, bem como suprime a produção de glicose endógena.

Apesar da maior parte da utilização da glicose periférica ocorrer no músculo( $85 \%)$ em comparação com o tecido adiposo, este último tem importante papel na homeostase glicêmica através da regulação da liberação de ácidos graxos a partir dos triglicerídeos armazenados. A insulina é um importante hormônio antilipolítico. Pequenas elevações de insulina são capazes de inibir a lipólise, levando a diminuição dos níveis plasmáticos de ácidos graxos ${ }^{8}$.

A via pela qual a glicose é oferecida ao organismo tem também papel importante. A glicose administrada através do sistema digestório tem potente efeito sobre a secreção de insulina se comparada com a administração venosa de glicose. Esse efeito aumentado está relacionado à liberação de GLP-1 e glucose-dependent insulinotropic polyptide(GIP) $)^{9,10}$.

1. Professor de Cirurgia da Universidade Estácio de Sá; Mestrando em Cirurgia pela Universidade Federal do Rio de Janeiro - UFRJ

2. Professor Adjunto do Departamento de Cirurgia da UFRJ - Chefe da Seção de Cirurgia Bariátrica do HUCFF.

Recebido em 20/10/2006

Aceito para publicação em 22/12/2006

Conflito de interesses: nenhum

Fonte de financiamento: nenhuma 
Modernas teorias admitem que o diabetes do tipo 2 é uma doença heterogênea, podendo ser causada tanto por resistência insulínica como por secreção de insulina inadequada pelas células beta pancreáticas.

Precocemente, na história natural do diabetes tipo 2, a resistência insulínica está bem estabelecida, progredindo posteriormente para intolerância à glicose e diabetes ${ }^{11}$. Por outro lado, estudos clínicos em humanos e animais já documentaram defeitos na função dessas mesmas células beta pancreáticas ${ }^{12}$.

\section{HORMÔNIOS GASTROINTESTINAIS}

A grelina é um hormônio produzido, quase que em sua totalidade, no fundo gástrico, área excluída após realização de by-pass gástrico em Y de Roux ${ }^{13}$. Esse peptídeo é o único hormônio entérico conhecido com efeito estimulante sobre o apetite ${ }^{14-16}$. Ocorre aumento dos níveis plasmáticos antes da alimentação com posterior queda, reforçando a hipótese da função orexígena desse hormônio ${ }^{17,18}$. Esse fato pode explicar os melhores resultados das técnicas que excluem ou ressecam o fundo do estômago quando comparadas com a banda gástrica. Não parece, no entanto, que esse hormônio tenha qualquer efeito direto sobre o controle do diabetes, que ocorre após as operações. Pode até mesmo ter efeito contrário, dificultando a liberação e ação da insulina ${ }^{19}$.

Alguns outros hormônios como o peptídeo YY(PYY) e o "glucagon-like peptide-1"(GLP-1), são produzidos na parte final do íleo mediante o estímulo ocasionado pela presença de alimento nessa região. As células endócrinas tipo L, monitoram o conteúdo no intestino delgado mais distal e no cólon. Elas liberam PYY em resposta à presença de bile, gordura, glicose e aminoácidos ${ }^{20}$.

O GLP-1 e o GIP, são denominados incretinas, uma vez que produzidos no sistema digestório, atuam diretamente sobre o pâncreas estimulando a secreção de insulina ${ }^{21}$. O GLP1 pode ainda, inibir o esvaziamento gástrico e consequentemente diminuir a ingestão de alimentos ${ }^{22}$.

Outros hormônios como a colecistoquinina, a gastrina, e a motilina, são produzidos no sistema digestório mas sua discussão tem pouco interesse no momento, uma vez que suas ações têm pouca relação com o potencial hormonal de controle do diabetes tipo 2 .

\section{A CIRURGIA BARIÁTRICA E O DIABETES TIPO 2}

De todas as doenças associadas à obesidade, aquela que apresenta melhor controle após cirurgia bariátrica é o diabetes do tipo $2^{23}$. Foram avaliados 3568 pacientes diabéticos submetidos ao by-pass gástrico em Y de Roux, evidenciou-se uma completa remissão da doença entre 82 a $98 \%$ dos pacientes $^{24-28}$. A cura da intolerância à glicose foi quase universal.

Em um estudo com pacientes obesos com intolerância à glicose operados e seguidos por aproximadamente cinco anos e meio, a cirurgia bariátrica foi capaz de diminuir o índice de progressão para o diabetes em mais de trinta vezes ${ }^{29}$. O controle da glicemia a longo prazo, bem como dos níveis de hemoglobina glicosilada também estão bem documentados em estudos que avaliaram resultados tardios, com tempo de acompanhamento superior a $14 \operatorname{anos}^{24}$.

O by-pass gástrico em Y de Roux é então um método extremamente eficiente de controle do diabetes tipo 2, doença tradicionalmente considerada progressiva. Esse tipo de operação é ainda o mais realizado em todo o mundo para tratamento da obesidade, segundo levantamento mundial realizado por Buchwald $^{30}$, superando as demais operações pela consistência nos resultados e poucas complicações à longo prazo.

\section{POSSÍVEIS MECANISMOS DE AÇÃO}

A realização de um by-pass gástrico em Y de Roux, produz em primeiro lugar, do ponto de vista mecânico, restrição gástrica. Essa capacidade gástrica restrita leva à saciedade precoce com conseqüente diminuição do volume das refeições. Todavia, se esse fosse o único mecanismo responsável, o sistema de homeostase energética provocaria um aumento compensatório na frequiência e quantidade calórica das refeições, o que sabidamente não acontece após esse tipo de procedimento $^{31}$.

O by-pass gástrico inclui ainda a exclusão do tubo digestivo proximal, pela realização da reconstrução em Y de Roux. Essa associação do fator de má absorção poderia então justificar a maior eficácia desse procedimento quando comparado aos procedimentos puramente restritivos. No entanto, má absorção clincamente significativa, medida através da dosagem plasmática de albumina e pré-albumina, bem como de gordura fecal, não é obeservada após esse tipo de procedimento $^{32-35}$

Devemos considerar então que os mecanismos hormonais estão envolvidos na perda de peso e no controle de doenças associadas, em especial o diabetes, após cirurgia bariátrica.

Apesar de todos os procedimentos bariátricos produzirem perda de peso, o by-pass gástrico em Y de Roux e as derivações bilio-pancreáticas são as mais eficientes tanto em relação à perda de peso como na melhora da homeostase glicêmica ${ }^{25,36}$. Ambos excluem o intestino proximal do trânsito alimentar, o que pode significar que essa região ativa do ponto de vista hormonal, pode ser importante no resultado final desses procedimentos ${ }^{37}$.

Um estudo experimental recente realizado por Rubino e Marescaux ${ }^{38}$, ratificou a hipótese do valor do intestino proximal no controle do diabetes. Outros autores com Pories ${ }^{39}$, acreditam no valor do intestino proximal na gênese do diabetes tipo 2, e que a exclusão desse segmento modificaria os sinais para o pâncreas das incretinas produzidas nessa região.

Outra hipótese se baseia no fato das cirurgias bariátricas, como o by-pass gástrico em Y de Roux e as derivações bilio-pancreáticas, permitirem a presença de alimentos mal digeridos de forma precoce no intestino delgado distal. A presença desses nutrientes no íleo diminui a motilidade gastrointestinal, o esvaziamento gástrico, a velocidade do trânsito desde a boca até o ceco e conseqüentemente a ingestão alimentar, mecanismo conhecido como freio ileal ${ }^{40}$. Esse meca- 
nismo neural seria mediado por hormônios produzidos no íleo como o PYY e o GLP-1 ${ }^{41-43}$. Segundo essa linha de raciocínio a melhora do diabetes após cirurgia bariátrica estaria relacionada ao intestino distal e não ao intestino proximal.

Para confirmar essa hipótese alguns autores propuseram a interposição de um segmento ileal no início do jejuno e obtiveram aumento na produção de PYY e GLP-1, reforçando a teoria do freio ileal ${ }^{44-46}$.

\section{ÁREAS DE PESQUISA}

Apesar de estarmos ampliando nosso conhecimento sobre o papel dos hormônios gastrointestinais no controle do apetite, da regulação do peso e do metabolismo, muitos fatos ainda carecem de melhores explicações.

Por que indivíduos obesos tem níveis diminuídos de alguns hormônios intestinais como o PYY? Quais são os resultados a longo prazo do tratamento cirúrgico em relação à modificação na produção dessses hormônios? Existem mutações genéticas que levam a essas alterações? Esse tipo de cirurgia ou modificações dessas técnicas podem ser utilizados em pacientes diabéticos tipo 2 não obesos mórbidos?

Sem dúvida essas e outras perguntas precisam ser respondidas e representam áreas de pesquisa atuais e de extrema relevância para o completo esclarecimento do papel dos hormônios intestinais na gênese e no controle do diabetes tipo 2 .

\begin{abstract}
Type 2 diabetes mellitus is an epidemic health problem. Approximately, 90\% of diabetic patients are overweight or are obese. The current increase in the prevalence of obesity has been associated with an increase in the prevalence of type 2 diabetes. Bariatric surgery is the most effective treatment for morbid obese patients in terms of controlling weight and co-morbidities. Sustained normal plasma concentration of glucose has been reported in most diabetic morbid obese patients, which has been managed surgically. Available data show a significant alteration in the production of some gastrointestinal hormones, which might explain the improvement of glucose metabolism following these procedures. Diabetic patient improvements following some bariatric surgeries seems to be an independent factor unrelated to the amount of weight loss. The authors reviewed data published on the effects of bariatric surgery in diabetic patient improvements and the possible mechanisms responsible for this control (Rev. Col. Bras. Cir. 2007; 34(5): 343-346).
\end{abstract}

Key words: Bariatric Surgery; Diabetes Mellitus, Type 2 ; Gastrointestinal Hormones.

\section{REFERÊNCIAS}

1. Narayan KM, Gregg EW, Fagot-Campagna A, Engelgau MM, Vinicor F. Diabetes: a common, growing, serious, costly, and potentially preventable public health problem. Diabetes Res Clin Pract. 2000;50(Suppl 2):S77-84.

2. Albu J, Pi-Sunyer FX. Obesity and diabetes. In Bray GA, Bouchard C, James WPT, editors. Handbook of obesity. New York: Marcel Dekker; 1998. p. 697-707.

3. Hu FB, Manson JE, Stampfer MJ, Colditz G, Liu S, Solomon CG, Willett WC. Diet, lifestyle and the risk of type 2 diabetes mellitus in women. N Engl J Med. 2001;345(11):790-7.

4. Le Roux CW, Bloom SR. Why do patients lose weight after Roux-en-Y gastric bypass? J Clin Endocrinol Metab. 2005:90(1):591-2.

5. Rubino F. Bariatric surgery: effects on glucose homeostasis. Curr Opin Clin Nutr Metab Care. 2006;9(4):497-507.

6. Rubino F, Gagner M, Gentileschi P, Kini S, Fukuyama S, Feng J, Diamond E. The early effect of the Roux-en-Y gastric bypass on hormones involved in body weight regulation and glucose metabolism. Ann Surg. 2004;240(2):236-42.

7. Grill V. A comparison of brain glucose metabolism in diabetes as measured by positron emission tomography or by arteriovenous techniques. Ann Med. 1990;22(3):171-6.

8. Groop LC, Bonadonna RC, Del Prato S, Ratheiser K, Zyck K, Ferrannini E, DeFronzo RA. Glucose and free fatty acid metabolism in non-insulin- dependent diabetes mellitus. Evidence for multiple sites of insulin resistance. J Clin Invest. 1989;84(1):205-13.

9. Drucker DJ. Glucagon-like peptides. Diabetes. 1998;47(2):15969.
10. Holst JJ, Gromada J, Nauck MA. The pathogenesis of NIDDM involves a defective expression of the GIP receptor. Diabetologia. 1997;40(8):984-6.

11. Groop L. Genetics of the metabolic syndrome. Br J Nutr. 2000;83(Suppl 1):S39-S48.

12. Cavaghan MK, Ehrmann DA, Polonsky KS. Interactions between insulin resistance and insulin secretion in the development of glucose intolerance. J Clin Invest. 2000;106(3):329-33.

13. Ariyasu H, Takaya K, Tagami T, Ogawa Y, Hosoda K, Akamizu T, Suda M, Koh T, Natsui K, Toyooka S, Shirakami G, Usui T, Shimatsu A, Doi K, Hosoda H, Kojima M, Kangawa K, Nakao $\mathrm{K}$. Stomach is a major source of circulating ghrelin, and feeding state determines plasma ghrelin-like immunoreactivity levels in humans. J Clin Endocrinol Metab. 2001;86(10):4753-8.

14. Kojima M, Hosoda H, Date Y, Nakazato M, Matsuo H, Kangawa $\mathrm{K}$. Ghrelin is a growth-hormone-releasing acylated peptide from stomach. Nature.1999;402(6762):656-60.

15. Nakazato M, Murakami N, Date Y, Kojima M, Matsuo H, Kangawa K, Matsukura S. A role for ghrelin in the central regulation of feeding. Nature. 2001;409(6817):194-8.

16. Wren AM, Small CJ, Ward HL, Murphy KG, Dakin CL, Taheri S, Kennedy AR, Roberts GH, Morgan DG, Ghatei MA, Bloom SR. The novel hypothalamic peptide ghrelin stimulates food intake and growth hormone secretion. Endocrinology. 2000;141(11):4325-8.

17. Cummings DE, Shannon MR. Roles for ghrelin in the regulation of appetite and body weight. Arch Surg. 2003;138(4):389-96.

18. Cummings DE, Purnell JQ, Frayo RS, Schmidova K, Wisse $\mathrm{BE}$, Weigle DS. A preprandial rise in plasma ghrelin levels suggests a role in meal initiation in humans. Diabetes. 2001;50(8):1714-9. 
19. Svensson J, Lönn L, Jansson JO, Murphy G, Wyss D, Krupa D, Cerchio K, Polvino W, Gertz B, Boseaus I, Sjöström L, Bengtsson BA. Two-month treatment of obese subjects with the oral growth hormone $(\mathrm{GH})$ secretagogue MK-677 increases GH secretion, fat-free mass, and energy expenditure. J Clin Endocrinol Metab. 1998;83(2):362-9.

20. Ballantyne GH. Peptide YY(1-36) and Peptide YY(3-36): Part I. Distribution, release and actions. Obes Surg. 2006;16(5):651-8.

21. Thompson JC, Townsend CM Jr. Endocrine pancreas. In: Townsend CM Jr. Sabiston textbook of surgery, $17^{\text {th }}$ ed. London: Saunders; 2004. p. 1001-21.

22. Drucker DJ. Enhancing incretin action for the treatment of type 2 diabetes. Diabetes Care. 2003;26(10):2929-40.

23. Cummings DE, Overduin J, Foster-Schubert KE. Gastric bypass for obesity: mechanisms of weight loss and diabetes resolution. J Clin Endocrinol Metab. 2004;89(6):2608-15.

24. Pories WJ, Swanson MS, MacDonald KG, Long SB, Morris PG, Brown BM, Barakat HA, deRamon RA, Israel G, Dolezal JM, et al. Who would have thought it? An operation proves to be the most effective therapy for adult-onset diabetes mellitus. Ann Surg. 1995;222(3):339-50; discussion 350-2.

25. Schauer PR, Burguera B, Ikramuddin S, Cottam D, Gourash W, Hamad G, Eid GM, Mattar S, Ramanathan R, Barinas-Mitchel E, Rao RH, Kuller L, Kelley D. Effect of laparoscopic Roux-en $\mathrm{Y}$ gastric bypass on type 2 diabetes mellitus. Ann Surg. 2003;23894):467-84; discussion 84-5.

26. Sugerman HJ, Wolfe LG, Sica DA, Clore JN. Diabetes and hypertension in severe obesity and effects of gastric bypassinduced weight loss. Ann Surg. 2003;237(6):751-6; discussion 757-8.

27. Wittgrove AC, Clark GW. Laparoscopic gastric bypass, Rouxen-Y- 500 patients: technique and results, with 3-60 month follow-up. Obes Surg. 2000;10(3):233-9.

28. Schauer PR, Ikramuddin S, Gourash W, Ramanathan R, Luketich J. Outcomes after laparoscopic Roux-en-Y gastric bypass for morbid obesity. Ann Surg. 2000;232(4):515-29.

29. Long SD, O’Brien K, MacDonald KG Jr, Leggett-Frazier N, Swanson MS, Pories WJ, Caro JF. Weight loss in severely obese subjects prevents the progression of impaired glucose tolerance to type II diabetes. A longitudinal interventional study. Diabetes Care. 1994;17(5):372-5.

30. Buchwald H, Williams SE. Bariatric surgery worldwide 2003. Obes Surg. 2004;14(9):1157-64.

31. Halmi KA, Mason E, Falk JR, Stunkard A. Appetitive behavior after gastric bypass for obesity. Int J Obes. 1981;5(5):457-64.

32. Brolin RE. Bariatric surgery and long-term control of morbid obesity. JAMA. 2002;288(22):2793-6.

33. Faraj M, Jones P, Sniderman AD, Cianflone K. Enhanced dietary fat clearance in postobese women. J Lipid Res. 2001;42(4):57180.

34. MacLean LD, Rhode BM, Nohr CW. Long- or short-limb gastric bypass? J Gastrointest Surg. 2001;5(5):525-30.
35. Näslund I. Gastric bypass versus gastroplasty. A prospective study of differences in two surgical procedures for morbid obesity. Acta Chir Scand. 1987; Suppl 536:1-60.

36. Greenway SE, Greenway FL, Klein S. Effects of obesity surgery on non-insulin-dependent diabetes mellitus. Arch Surg. 2002;137(10):1109-17.

37. Hickey MS, Pories WJ, MacDonald KG Jr, Cory KA, Dohm GL, Swanson MS, Israel RG, Barakat HA, Considine RV, Caro JF, Houmard JA. A new paradigm for type 2 diabetes mellitus: could it be a disease of the foregut? Ann Surg. 1998;227(5):63743; discussion 643-4.

38. Rubino F, Marescaux J. Effect of duodenal-jejunal exclusion in a non-obese animal model of type 2 diabetes: a new perspective for an old disease. Ann Surg. 2004;239(1):1-11.

39. Pories WJ, Albrecht RJ. Etiology of type II diabetes mellitus: role of the foregut. World J Surg. 2001;25(4):527-31. Epub 2001 Apr 18.

40. Spiller RC, Trotman IF Higgins BE, Ghatei MA, Grimble GK, Lee YC, Bloom SR, Misiewicz JJ, Silk DB. The ileal brakeinhibition of jejunal motility after ileal fat perfusion in man. Gut. 1984;25(4):365-74.

41. Lin HC, Zhao XT, Wang L, Wong H. Fat-induced ileal brake in the dog depends on peptide YY. Gastroenterology. 1996;110(5):1491-5.

42. Cuche G, Cuber JC, Malbert CH. Ileal short-chain fatty acids inhibit gastric motility by humoral pathway. Am J Physiol Gastrointest Liver Physiol. 2000;279(5):G925-30.

43. Rubino F, Gagner M. Potential of surgery for curing type 2 diabetes mellitus. Ann Surg. 2002;236(5):554-9.

44. Koopmans HS, Ferri GL, Sarson DL, Polak JM, Bloom SR. The effects of ileal transposition and jejunoileal bypass on food intake and GI hormone levels in rats. Physiol Behav. 1984;33(4):601-9.

45. Ueno T, Shibata C, Naito H, Jin XL, Funayama Y, Fukushima K, Matsuno S, Sasaki I. Ileojejunal transposition delays gastric emptying and decreases fecal water content in dogs with total colectomy. Dis Colon Rectum. 2004;45(1):109-16; discussion 116-8.

46. Ohtani N, Sasaki I, Naito H, Shibata C, Tsuchiya T, Matsuno S. Effect of ileojejunal transposition of gastrointestinal motility, gastric emptying, and small intestinal transit in dogs. J Gastrointest Surg. 1999; 3(5):516-23.

Como citar este artigo:

Martins MVD, Souza AAP. Mecanismos cirúrgicos de controle do diabetes mellitus tipo 2 após cirurgia bariátrica. Rev Col Bras Cir. [periódico na Internet] 2007 Set-Out; 34(5). Disponível em URL: www.scielo.br/rcbc

Endereço para correspondência:

Marcus Vinicius Dantas de Campos Martins

Av. Armando Lombardi 1000 bloco 2 sala 214

Barra da Tijuca

22640-000 - Rio de Janeiro - RJ

E-mail:mvdantas@hotmail.com.br 